\title{
Über ein eindimensionales Modell der finiten Elastostatik
}

\author{
A. LANGENBACH
}

The deformation of a rod $S=[0,1]$ is given by a diffeomorphism $w: S \rightarrow S_{w}=[0, w(1)]$ from the set $\left\{w \in W_{2}^{2}(0,1): w(0)=0, w:(s) ; 0\right.$ ( $\left.\left.s \in S\right)\right\}$. The function $1 n w^{\prime}$ appears as ineasure of deformation. The function $w$ is solution to a second order ordinary differential equation with the second boundary condition $w^{\prime}(1)=p \in \mathbb{R}^{+}$. By an open-and-closed argument we show that the set of those $p$ for which the boundary problem is uniquely solvable, is all $\mathbb{R}^{+}$.

Key words: finite elasticity, boundary value problems, ordinary differential equations AMS subject classification: $47 \mathrm{EO}, 73005$

\section{Endliche Deformation eines eingebetteten Zugstabes, Formulierung der Aufgabe}

Wir betrachten ein mathematisches Modell der in Abb. 1 skizzierten Situation. Ein elastischer. Stab wird durch Druck- bzw. Zugkräfte gestaucht oder gedehnt. Der Verschiebung des Stabes wirkt das anhaftende äußere Medium entgegen.

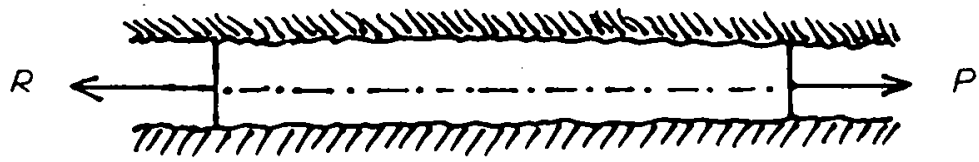

Abb.1

Wie wählen folgende Bezeichnungen: $S=[0,1]$ bezeichnet das dem unbelasteten Stab zugeordnete Intervall. Die Funktion $w: S \rightarrow w(S), w \in C^{1}(S)$, beschreibt die Verformung des Stabes, $w(s)=: x \in w(S)=: S_{w}$. Zur Gewährleistung einer Homöomorphie zwischen $S$ und $S_{w}$ fordern wir $w^{\prime}(s)>0$ für $s \in S$. Mit $v_{w} \in C^{1}\left(S_{w}\right)$ bezeichnen wir eine virtuelle Verschiebung des deformierten Stabes, mit $T_{w}: S_{w} \rightarrow \mathbf{R}$ die im Stab auftretenden Druck - bzw. Zugspannungen. Dann ist $\partial A_{0}=\int_{S_{w}} T_{w}(x) v_{w}^{\prime}(x) d x$ die virtuelle Arbeit der elastischen Kräfte des Stabes. Die virtuelle Arbeit der äußeren Kräfte setzt sich aus den Anteilen $\partial A_{1}=P v_{w}(w(1))-R v_{w}(w(0))$, der Arbeit der Kräfte $P$ und $R$, sowie $\partial A_{2}=-\int_{S_{w}} Q(x) v_{w}(x) d x$, der Arbeit des Widerstand leistenden anhaftenden Me diums zusammen. Die Gleichgewichtsbedingung (Stabgleichung) lautet in Anwendung des Prinzips der virtuellen Verschiebungen

$$
\delta A_{0}=\delta A_{1}+\delta A_{2} \text { für alle } v_{w} \in C^{1}\left(S_{w}\right) .
$$

Als Referenzkonfiguration wählen wir $S_{\text {id }}=S$. Die Beziehung

$$
\left(L v_{\mathrm{w}}\right)(s):=v_{\mathrm{id}}(s):=v_{\mathrm{w}}(w(s)) \quad(s \in S)
$$


definiert einen Isomorphismus zwischen den Räumen virtueller Verschiebungen $C^{1}\left(S_{w}\right)$ und $C^{1}(S)$. Durch Differentiation erhalten wir

$$
v_{\mathrm{id}}(s)=v_{\mathrm{w}}(w(s)) w^{\prime}(s) \quad(s \in S) .
$$

Wir können damit die Gleichgewichtsbedingung (1.1) auf die Referenzkonfiguration umschreiben. Es ist nach (1.2), (1.3)

$$
\begin{aligned}
0 & =\delta A_{0}-\delta A_{1}-\delta A_{2} \\
& =\int_{S_{w}}\left\{T_{w}(x) v_{w}^{\prime}(x)+Q(x) v_{w}(x)\right\} d x-P v_{w}(w(1))+R v_{w}(w(0)) . \\
& =\int_{S}\left\{T_{w}(w(s)) v_{i d}(s)+Q(w(s)) v_{i d}(s) w^{\prime}(s)\right\} d s-P v_{i d}(1)+R v_{i d}(0) .
\end{aligned}
$$

Zur vollständigen Beschreibung der angenommenen Situation benötigen wir noch zwei Materialgesetze. Aus der Variationsgleichung (1.4) können wir unter bekannten Stetigkeitsannahmen die Differentialgleichung

$$
-d T_{w}(w(s)) / d s+Q(w(s)) w^{\prime}(s)=0 \quad(s \in S)
$$

und die statischen Randbedingungen $T_{w}(w(1))=P, T_{w}(w(0))=R$ gewinnen. Nehmen wir das Elastizitätsgesetz $T_{w}(w(s))=\sigma\left(w^{\prime}(s)\right)(s \in S)$ an, so erhalten wir unter Beruicksichtigung der Annahme $w^{\prime}(s)>0(s \in S)$ aus (1.S) die Differentialgleichung

$$
-\sigma^{\prime}\left(w^{\prime}(s)\right) w^{\prime \prime}(s) / W^{\prime}(s)+Q(w(s))=0 \quad(s \in S) .
$$

Die Materialfunktion $\sigma=\sigma(t)$ nehmen wir als stetig differenzierbar mit $\sigma^{\prime}(t)>0(t>0)$ und $\sigma(1)=0$ an. Für die ebenfalls stetig differenzierbare Umkehrfunktion gelte $\sigma^{-1}: \mathbf{R} \rightarrow$ $(0, \infty)$. Wir setzen nun $a(t)=\int_{0}^{t} \sigma^{\prime}\left(e^{y}\right) d y(t \in \mathbb{R})$ und nehmen die Rückstellkraft mit $Q(w(s))=q(s, w(s)-s)(s \in S)$ an, in $\operatorname{der} q: S \times \mathbb{R} \rightarrow \mathbb{R}$ eine Carathéodory-Funktion sei; es gelte $q(s, 0)=0$ und $q(s, \cdot)$ sei monoton wachsend für fast alle $s \in S$. Mit $a^{\prime}(\ln t)$ $=\sigma^{\circ}(t)(t>0)$ nimmt die Gleichung (1.6) die Form

$$
-d a\left(\ln w^{\prime}(s)\right) / d s+q(s, w(s)-s)=0 \quad(s \in S)
$$

an. Die Funktion $a=a(t)$ ist stetig differenzierbar mit $a^{\prime}(t)>0(t \in \mathbb{R})$ und $a(0)=0$. Gehen wir mit dem Elastizitätsgesetz in die statischen Randbedingungen ein, so erhalten wir z.B. $w^{\prime}(1)=\sigma^{-1}(P)=: p$, wobei die Funktion $p=p(P)$ stetig differenzierbar mit $p^{\prime}(P)$ $>0(P \in R)$ und $p(0)=1$ ist.

In Anlehnung an das betrachtete Beispiel einer eindimensionalen Aufgabe der finiten Elastizitätstheorie betrachten wir in naheliegender Verallgemeinerung die folgende repräsentative Randwertaufgabe :

Gesucht ist eine Lösung $w \in W_{2}^{2}\left(S^{\circ}\right)$ der Differentialgleichung

$$
\text { - } d a\left(s, \ln w^{\prime}(s)\right) / d s+q(s, w(s)-s)=0 \text { f.f.a. } s \in S,
$$

die der Randbedingung

$$
w(0)=0, w^{\prime}(1)=p
$$

genügt. Dabei sei $S^{\prime}:=(0,1)$; der Sobolew-Raum $W_{2}^{2}\left(S^{\prime}\right)$ ist stetig in den Raum $C^{1}(S)$ 
eingebettet und kann als Raum stetiger Funktionen mit absolutstetiger Ableitung betrachtet werden.

Die Gleichung (1.8) kann einerseits als eindimensionales Analogon der allgemeinen Gleichungen der finiten Elastostatik gedeutet werden (vgl. [3]), enthält andererseits die Gleichung (1.7) des̉ eingangs betrachteten Modells eines eingebetteten Zugstabes als Spezialfall. Dabei fallen jedoch Besonderheiten gegenuber einem "formalen" eindimensionalen Modell auf:

1. Die durch den Logarithmus vermittelte spezielle Abhängigkeit der Spannungen vom Deformationsmaß weist auf unterschiedliche Reaktion des Materials bei Druckbzw. Zugbelastungen hin.

2. Die durch die Rückstellkraft des anhaftenden äußeren Mediums vermittelte "Volumenkraft" $q\left(s, w\left(s^{\prime}\right)-s\right)$ ( $s \in S$ ) hängt sowohl von der "Euler-Variablen" $x$ wie auch von der "Lagrange-Variablen" $s$ ab, fällt also nicht unter die häufig vorausgesetzten Lagrange-Kräfte (dead loads) oder Euler-Kräfte (Potentiale).

Die Randbedingungen (1.9) gehören zu den sog. "gemischten" Randbedingungen, die im allgemeinen dreidimensionalen Fall erhebliche analytische Schwierigkeiten verursachen. Wir wollen zeigen, daß die Aufgabe (1.8); (1.9) unter physikalisch akzeptablen Bedingungen für beliebige endliche Zug- und Druckkräfte eindeutig bestimmte Lösungen besitzt. Dabei interessiert das Modell selbst nur mittelbar. Bekanntlich werden die Gleichgewichtsbedingungen in der finiten Elastostatik - im Unterschied zur klassischen Elastizitätstheorie - auf den deformierten Körper bezogen (vgl. [3]), in unserem Beispiel also auf $S_{w}$ und nicht auf $S$. Für die üblichen dreidimensionalen Probleme der finiten Elastostatik gibt es keine globalen Resultate, sie sind im allgemeinen auch nicht zu erwarten.

Anliegen dieser Arbeit ist der Nachweis einer globalen Lösung in einem physikalisch motivierten Spezialfall mit funktionalanalytischen Hilfsmitteln. Die Nebenbedingung $w^{\prime}(s)>0$, die einen Homöomorphismus bei der Verformung des Stabes garantieren muß, ist nichtlinear und verweist auf ein Problem der globalen Analysis. Als Lösungsverfahren dient uns eine nichttriviale Variante des Satzes über implizite Funktionen, die in Verbindung mit Regularitätsresultaten und der topologischen Betrachtung des Zusammenhangs (der zugelassenen Zugkräfte) zu einem globalen Hilfsmittel wird. Man kann erwarten, daß dieser Zugang auch in anderen Aufgaben der finiten Elastostatik zum Erfolg fuihrt.

Die Aufgabe (1.8),(1.9) erfordert eine Nebenbedingung. Es sei

$$
W^{+}:=\left\{w \in W_{2}^{2}\left(S^{\prime}\right): W^{\prime}(s)>0 \quad(s \in S)\right\} \text { und zusätzlich } W \in W^{+}
$$

gefordert. Die Funktionen $a, q: S \times \mathbb{R} \rightarrow \mathbb{R}$ sollen speziell die im betrachteten Beispiel geforderten Eigenschaften besitzen. Wir fordern daher folgendes $\left(q_{0} \in L_{2}\left(S^{\prime}\right)\right)$ :

$\left(\mathbf{A}_{1}\right)$ a $\in C^{2}(S \times \mathbf{R}), a(s, 0)=0$ für $s \in S$.

$\left(\mathrm{A}_{2}\right)\left(\partial_{a} / \partial t\right)(s, t)>0$ für $(s, t) \in S \times \mathbb{R}$.

$\left(Q_{1}\right) \quad q: S \times \mathbb{R} \rightarrow \mathbb{R}$ ist Carathéodory-Funktion, $q(s, 0)=0$ f.f.a. $s \in S$.

$\left(Q_{2}\right)\left[q\left(s, t_{1}\right)-q\left(s, t_{2}\right)\right]\left(t_{1}-t_{2}\right) \geq 0$ f.f.a. $s \in S$ und alle $t_{1}, t_{2} \in \mathbb{R}$.

$\left(Q_{3}\right)\left|q\left(s, t_{1}\right)-q\left(s, t_{2}\right)\right| \leq q_{0}(s)\left|t_{1}-t_{2}\right|$ f.f.a. $s \in S$ und alle $t_{1}, t_{2} \in \mathbb{R}$. 
Zur. Lösung des Randwertproblems (1.8), (1.9) für beliebige Parameter $p$ aus einer Umgebung $U(1)$ benutzen wir den Satz ubber Lipschitz-stetige implizite Funktionen aus der Arbeit [1]. Wir bemerken dazu, daß die Menge $W^{+}$offen in $W_{2}^{2}\left(S^{\prime}\right)$ und die Funktion $w_{0}=$ id eine Lösung des Randwertproblems (1.8), (1.9) fur den Parameter $p=1$ ist. Zur Formulierung einer Operatorgleichung für die Aufgabe (1.8), (1.9) benötigen wir die Sobolew-Räume $W_{2}^{2}\left(S^{\prime}\right) \subset W_{2}^{1}\left(S^{\prime}\right) \subset C(S)$ mit jeweils stetiger Einbettung. Es sei noch $D\left(S^{\prime}\right)$ der Raum der $C^{\infty}$ - Funktionen mit kompakten Träger in $S^{\prime}, D^{+}:=\left\{\sigma \in W_{2}^{1}\left(S^{\prime}\right): \sigma(s)>\right.$ $0(s \in S)\}$.

Bemerkung 1.1: Mit $\sigma^{\prime} \in L_{1}\left(S^{\prime}\right)$ bezeichnen wir die Distributionsableitung einer Funktion $\sigma \in L_{1}\left(S^{\prime}\right): \int_{S} \sigma h^{\prime} d s=-\int_{S} \sigma^{\prime} h d s$ fur alle $h \in D\left(S^{\prime}\right)$. Es ist $\sigma^{\prime}$ genau dann Distributionsableitung von $\sigma$, wenn $\sigma$ absolutstetig ist, und es gilt $\sigma^{\circ}(s)=d \sigma(s) / d s$ f.f.a. $s \in S$. Im folgenden formulieren wir ohne Beweis einige Eigenschaften von Superpositionsoperatoren. Lemma 1.1 und Satz 1.1 sind einfache Adaptionen einiger Ergebnisse von T. VALENT [3].

Bemerkung 1.2 : Für $\sigma \in D^{+}$ist $\ln \sigma \in W_{2}^{1}\left(S^{\prime}\right)$ und es gilt $(\ln \sigma)^{\prime}=\sigma^{\prime} \%$. Die Abbildung $\ln : D^{+} \rightarrow W_{2}^{1}\left(S^{\circ}\right)$ ist stetig.

Lemma 1.1: Für a $\in C^{1}(S \times \mathbf{R})$ ist der Operator

$A: W_{2}^{1}\left(S^{\prime}\right) \rightarrow W_{2}^{1}\left(S^{\prime}\right),(A \sigma)(s)=a(s, \sigma(s)) \quad(s \in S)$

stetig. Mit

$$
\left(A_{s} \sigma\right)(s)=(\partial a / \partial s)(s, \sigma(s)),\left(A_{t} \sigma\right)(s)=(\partial a / \partial t)(s, \sigma(s))
$$

für $a=a(s, t)((s, t) \in S \times \mathbb{R})$ gilt $A_{s} \sigma, A_{t} \sigma \in C(S)$ für

$\sigma \in W_{2}^{1}\left(S^{\prime}\right)$ und $d(A \sigma)(s) / d s=\left(A_{s} \sigma\right)(s)+\sigma^{\prime}(s)\left(A_{t} \sigma\right)(s)$ f.f.a. $s \in S$.

Satz 1.1 : Unter der Bedingung $\left(\mathrm{A}_{1}\right)$ ist der Operator $A$ in (1.11) stetig differenzierbar und es gilt $A^{\prime}(\sigma) \tau=\tau A_{t} \sigma$ für $\sigma, \tau \in W_{2}^{1}\left(S^{\prime}\right), A 0=0$.

Folgerung 1.1 : Die Funktion a: $S \times \mathbb{R} \rightarrow \mathbb{R}$ genüge der Bedingung $\left(\mathrm{A}_{1}\right)$. Dann ist der Operator

$\mathfrak{H}: W^{+} \rightarrow L_{2}\left(S^{\prime}\right),(\mathfrak{U} w)(s)=-d a\left(s, \ln w^{\prime}(s)\right) / d s$ f.f.a. $s \in S$

stetig differenzierbar und

$$
\mathscr{2}^{\prime}(w)_{v}=-\left[A_{t}\left(\ln w^{\prime}\right)\left(v^{\prime} / w^{\prime}\right)\right] \quad\left(w \in W^{+}, v \in W_{2}^{2}\left(S^{\prime}\right)\right) .
$$

Beweis : $Z u w \in W^{+}$finden wir ein $\delta>0$ derart, daß aus $\|v\|_{W_{2}^{2}}<\delta$ stets $w+v \in W^{+}$ folgt. Für solche $v$ ist

$$
\left\|\mathfrak{A}(w+v)-\mathfrak{U} w+\left[A_{t}\left(\ln w^{\prime}\right)\left(v^{\prime} / w^{\prime}\right)\right]^{\prime}\right\|_{L_{2}} \leq V(w, v)\|v\|_{W_{2}^{2}}
$$

mit

$$
V(w, v)=\left\|A\left(\ln \left(w^{\prime}+v^{\prime}\right)\right)-A\left(\ln w^{\prime}\right)-A_{t}\left(\ln w^{\prime}\right)\left(v^{\prime} / w^{\prime}\right)\right\|_{W_{2}^{1}} /\left\|v^{\prime}\right\|_{W_{2}^{1}}
$$


Auf der Menge $D^{+}$betrachten wir zunächst den Operator $\ln : D^{+} \rightarrow W_{2}^{1}\left(S^{\prime}\right)$ aus der Bemerkung 1.2. Dieser ist stetig differenzierbar und

$$
\ln ^{\prime}(\sigma) \tau=\tau / \sigma\left(\sigma \in D^{+}, \tau \in W_{2}^{1}\left(S^{\prime}\right)\right), \ln ^{\prime}(\sigma) \in \mathbb{R}\left(W_{2}^{1}\left(S^{\prime}\right) ; W_{2}^{1}\left(S^{\prime}\right)\right) \text {. }
$$

Nach Satz 1.1 ist dann der Operator

$$
A \ln : D^{+} \rightarrow W_{2}^{1}\left(S^{\circ}\right),(A \ln \sigma)(s)=a(s, \ln \sigma(s)) \quad(s \in S)
$$

stetig differenzierbar und es gilt

$$
(A \ln )^{\prime}(\sigma) \tau=A^{\prime}(\ln \sigma) \ln ^{\prime}(\sigma) \tau=(\tau / \sigma) A_{t}(\ln \sigma) .
$$

Für $w \in W^{+}$ist nun $\sigma:=W^{\prime} \in D^{+}$. Setzen wir dieses $\sigma$ mit $\tau:=v^{\prime} \in W_{2}^{1}\left(S^{\prime}\right)$ in (1.13) ein, so ergibt sich $V(w, v) \rightarrow 0$ bei $\|v\|_{W_{2}^{2}} \rightarrow 0$, woraus die Formel (1.12) folgt. Für $w_{n}, w \in$ $W^{+}$mit $\left\|w_{n}=w\right\|_{W_{2}^{2}} \rightarrow .0$ erhalten wir aus der Algebra-Eigenschaft von $W_{2}^{1}\left(S^{\prime}\right)$ fur ein $x>0$ die Abschätzung

$$
\left\|\mathfrak{U}^{\prime}\left(w_{n}\right) v-\mathfrak{I}^{\prime}(w) v\right\|_{L_{2}} \leq x\left\|A_{t}\left(\ln w_{n}^{\prime}\right) / w_{n}^{\prime}-A_{t}\left(\ln w^{\prime}\right) / w^{\prime}\right\| W_{2}^{1}\|v\|_{W_{2}^{2}}
$$

und folglich $\left\|\mathfrak{H}^{\prime}\left(w_{n}\right)-\mathfrak{H}^{\prime}(W)\right\| \rightarrow 0$, da die Operatoren $A_{t}$, ln $: W_{2}^{1}\left(S^{\prime}\right) \rightarrow W_{2}^{1}\left(S^{\prime}\right)$ stetig sind

Lemma 1.2 : Unter den Bedingungen $\left(\mathrm{Q}_{1}\right),\left(\mathrm{Q}_{3}\right)$ ist der Operator

$$
\$: W_{2}^{1}\left(S^{\prime}\right) \rightarrow L_{2}\left(S^{\prime}\right),(\$ w)(s)=q(s, w(s)-s) \quad(s \in S)
$$

\section{Lipschitz-stetig.}

Beweis : Mit der stetigen Einbettung $W_{2}^{1}\left(S^{\prime}\right) \subset C(S),\|w\|_{C} \leq x\|w\|_{W_{2}^{1}}$ für $w \epsilon$ $W_{2}^{1}\left(S^{\prime}\right)$, ist die Funktion $q(s, w(s)-s)(s \in S)$ meßbar und

$$
\begin{aligned}
\left\|\Re w_{1}-\Re w_{2}\right\|_{L_{2}}^{2} & =\int_{S}\left[q\left(s, w_{1}(s)-s\right)-q\left(s, w_{2}(s)-s\right)\right]^{2} d s \\
& s \int_{S}\left[q_{0}(s)\right]^{2}\left(w_{1}(s)-w_{2}(s)\right)^{2} d s s x^{2}\left\|w_{1}-w_{2}\right\| w_{2}^{2}\left\|q_{0}\right\|_{L_{2}}^{2}
\end{aligned}
$$

Die Randwertaufgabe (1.8) - (1.10) formulieren wir als Operatorgleichung in der folgenden Weise :

Gesucht ist ein Element $w \in W_{0}^{+}:=\left\{w \in W^{+}: w(0)=0\right\}$, welches der Gleichung

$$
\mathfrak{F}(w)-f(p)=0
$$

genügt. Dabei ist $p \in \mathbb{R}^{+}:=\{r \in \mathbb{R}: r>0\}, \mathfrak{F}: W_{0}^{+} \rightarrow L_{2} \times \mathbf{R}, \operatorname{pr}_{1} \mathfrak{F}:=\mathfrak{U}+\mathfrak{R}, \operatorname{pr}_{2} \mathfrak{F}(w)$ $:=w^{\prime}(1), \operatorname{pr}_{1} f(p)=0$ und $\operatorname{pr}_{2} f(p)=p$.

Unitatssatz 1.2 : Unter den Bedingungen $\left(A_{1}\right),\left(A_{2}\right),\left(Q_{1}\right)-\left(Q_{3}\right)$ besitzt die Gleichung (1.14) höchstens eine Lösung $w \in W_{0}^{+}$.

Beweis : Im gegenteiligen Fall haben wir Elemente $w_{1}, w_{2} \in W_{0}^{+}$mit

$0=\int_{S}\left\{\left[a\left(s, \ln w_{1}(s)\right)-a\left(s, \ln w_{2}^{\prime}(s)\right)\right]\left(w_{1}^{\prime}(s)-w_{2}^{\prime}(s)\right)\right.$ 


$$
\left.+\left[q\left(s, w_{1}(s)-s\right)-q\left(s, w_{2}(s)-s\right)\right]\left(w_{1}(s)-w_{2}(s)\right)\right\} d s .
$$

Für beliebige $\left(s, z_{1}\right),\left(s, z_{2}\right) \in S \times \mathbb{R}^{+}$finden wir

$$
a\left(s, \ln z_{1}\right)-a\left(s, \ln z_{2}\right)=\int_{0}^{1} \frac{(\partial a / \partial t)\left(s, \ln \left(z_{2}+\xi\left(z_{1}-z_{2}\right)\right)\right.}{z_{2}+\xi\left(z_{1}-z_{2}\right)} d \xi \cdot\left(z_{1}-z_{2}\right) .
$$

Aus der Eigenschaft $\left(\mathrm{A}_{2}\right)$ folgern wir nun die Existenz einer Konstanten $\mu_{0}>0$ mit $\mathrm{O}_{2}$ $\int_{S} H_{0}\left(w_{1}^{\prime}(s)-w_{2}^{\prime}(s)\right)^{2} d s$ und folglich ist $w_{1}(s)=w_{2}(s)(s \in S)$

\section{Lokale Ergebnisse}

Wir definieren die Menge $\mathfrak{M} \subset \mathbb{R}^{+}$mit Hilfe von Lösungen der Gleichung (1.14):

$$
\mathfrak{M}:=\left\{p \in \mathbb{R}^{+}: \mathfrak{F}(w)-f(p)=0 \text { für ein } w \in W_{\circ}^{+}\right\} .
$$

Es ist $\mathfrak{M} \neq \Phi$, denn zu $p=1$ ist $\mathfrak{F}($ id $)-f(1)=0$, also $1 \in \mathfrak{M}$.

Satz 2.1: Unter den Bedingungen des Unitätssatzes 1.2 ist $\mathfrak{M}$ offen.

Beweis : Zu beliebig fixiertem $p_{0} \in \mathfrak{M}$ gibt es nach (2.1) ein $w_{0} \in W_{0}^{+}$mit $\mathfrak{F}\left(w_{0}\right)$ - $f\left(p_{0}\right)=0$. Wir betrachten diese Beziehung als Ausgangspunkt zur Anwendung von Satz 4 der Arbeit [1] uiber Lipschitz-stetige implizite Funktionen. Wir setzen noch $H_{0}^{l}=\left\{u \in W_{2}^{l}\left(S^{\prime}\right): u(0)=0\right\}$ für $l=1,2$. Die Gleichung $\mathfrak{B}(x, z):=\mathfrak{F}(x)-f(z)=0$ betrachten wir für B : $U\left(w_{0}\right) \times V\left(p_{0}\right) \rightarrow L_{2}\left(S^{\prime}\right) \times \mathbf{R}$ mit $U\left(w_{0}\right)=W_{0}^{+} \subset H_{0}^{2}, V\left(p_{0}\right)$ $=\mathbb{R}^{+}$. Zerlegen wir $\mathfrak{G}(x, z)=F(x, z)+K(z) x$ mit $F(x, z)=\left(\mathfrak{U} x, x^{\prime}(1)-z\right)$ und $K(z) x$ $=(\$ x, 0)$, so besitzt $F$ nach Folgerung 1.1 die stetige partielle Fréchet-Ableitung $\partial F / \partial x: U\left(w_{0}\right) \times V\left(p_{0}\right) \rightarrow \mathfrak{R}\left(H_{0}^{2}, L_{2}\left(S^{\prime}\right) \times \mathbf{R}\right)$, während $K(z) \in \operatorname{Lip}\left(H_{0}^{2}, L_{2}\left(S^{\prime}\right) \times \mathbb{R}\right)$ von $z$ unabhängig ist. Wir zeigen noch die Inklusion $\left[(\partial F / \partial x)\left(w_{0}, p_{0}\right)\right]^{-1} \in \operatorname{Lip}\left(L_{2}\left(S^{\prime}\right)\right.$ $\left.\times \mathbb{R}, H_{o}^{2}\right)$ und bestätigen damit die Existenz einer Kugel $\mathfrak{R}\left(p_{0}, r_{1}\right) \subset \mathfrak{M}$. Wir haben also das Randwertproblem

$$
(\partial F / \partial x)\left(w_{0}, p_{0}\right) u+K\left(p_{0}\right) u=(f, p) \text { für beliebige }(f, p) \in L_{2}\left(S^{\prime}\right) \times \mathbf{R}
$$

zu lösen. Wenn wir die Operatoren $F, K$ einsetzen, so erhalten wir die Gleichung

$$
\left(\Omega_{0} u\right)(s):=-\frac{d}{d s}\left[\frac{\partial a}{\partial t}\left(s, \ln w_{0}^{\prime}(s)\right) \frac{u^{\prime}(s)}{w_{0}^{\prime}(s)}\right]+q(s, u(s)-s)=f(s)
$$

f.f.a. $s \in S$ und die Randbedingung

$$
u^{\prime}(1)=p
$$

für $u \in H_{0}^{2}$. Wir führen noch die Funktion

$$
P(s)=(\partial a / \partial t)\left(s, \cdot \ln w_{0}^{\prime}(s)\right) / w_{0}^{\prime}(s) \quad(s \in S)
$$

ein. Für $w_{0} \in W_{0}^{+}$ist $w_{0} \in D^{+}, \ln w_{0} \in W_{2}^{1}\left(S^{\prime}\right)$, und nach Lemma 1.1 unter den Bedingungen $\left(\mathrm{A}_{1}\right),\left(\mathrm{A}_{2}\right)$ gilt $P \in W_{2}^{1}\left(S^{\prime}\right), P(s) \geq P_{0}>0(s \in S)$, folglich $\mathbb{B}_{0}: H_{0}^{2} \rightarrow L_{2}\left(S^{\prime}\right)$. Zur Bestimmung einer schwachen Lösung des Randwertproblems (2.2), (2.3) definieren wir 
den Operator

$$
L_{0}: H_{0}^{1} \rightarrow\left(H_{0}^{1}\right)^{*},\left\langle L_{0} u, h\right\rangle=\int_{S}\left\{P(s) u^{\prime}(s) h^{\prime}(s)+q(s, u(s)-s) h(s)\right\} d s .
$$

Unter den Bedingungen $\left(A_{1}\right),\left(A_{2}\right),\left(Q_{1}\right)-\left(Q_{3}\right)$ ist $L_{0}$ stark monoton und Lipschitz-stetig. Ist nun $u \in H_{0}^{2}$ Lösung des Randwertproblems (2.2), (2.3), so gilt

$$
\left\langle L_{0} u, h\right\rangle=\int_{S} f(s) h(s) d s+P(1) p h(1)=:\left\langle f^{*}, h\right\rangle \quad\left(h \in H_{0}^{1}\right) .
$$

Dabei ist $f^{*} \in\left(H_{0}^{1}\right)^{*}$. Der inverse Operator $L_{0}^{-1}:\left(H_{0}^{1}\right)^{*} \rightarrow H_{0}^{1}$ ist Lipschitz-stetig (vgl. - [2: Satz 5.11] bei Identifizierung von $H_{0}^{1}$ und $\left.\left(H_{0}^{1}\right)^{*}\right)$. Sei $L_{0}^{-1} f^{*}=: u(f, p)$. Für $\left(f_{\nu}, p_{\nu}\right)$ $\in L_{2}\left(S^{\prime}\right) \times \mathbb{R}(\nu=1,2)$ erhalten wir die Abschätzung

$$
\begin{aligned}
\left\|u\left(f_{1}, p_{1}\right)-u\left(f_{2}, p_{2}\right)\right\|_{W_{2}^{1}} & \leq \text { const }\left\{\left\|f_{1}-f_{2}\right\|_{L_{2}}+\left|\rho_{1}-p_{2}\right|\right\} \\
& =\text { const }\left\|\left(f_{1}, p_{1}\right)-\left(f_{2}, p_{2}\right)\right\|_{L_{2} \times \mathbb{R}} .
\end{aligned}
$$

Speziell für $h \in D\left(S^{\prime}\right)$ folgern wir aus $(2.4)$

$$
\int_{S}\left\{P(s) u_{v}(s)-\int_{0}^{s}\left[q\left(\tau, u_{v}(\tau)-\tau\right)-f_{v}(\tau)\right] d \tau\right\} h^{\prime}(s) d s=0 \text { für } v=1,2
$$

und weiter mit geeigneten Konstanten $x_{v}$

$$
P(s) u_{v}(s)=\int_{0}^{s}\left[q\left(\tau, u_{v}(\tau)-\tau\right)-f_{v}(\tau)\right] d \tau+x_{\nu} \quad(s \in S) .
$$

Wir ersehen daraus, daß $\dot{u}_{v} \in H_{\mathrm{o}}^{2}$ die Gleichung (2.2) mit. $f=f_{\nu}$ und die Randbedingung (2.3) mit $p=p_{v}$ erfüllt. Schließlich gewinnen wir durch Differentiation aus (2.6). die Abschätzung

$$
\left\|u_{1}^{\prime \prime}-u_{2}^{\prime \prime}\right\|_{L_{2}} \text { s const. }\left\{\left\|u_{1}-u_{2}\right\|_{w_{2}^{1}}+\left\|f_{1}-f_{2}\right\|_{L_{2}}+\left|p_{1}-p_{2}\right|\right\}
$$

und mit (2.5) die Inklusion $\left[(\partial F / \partial x)\left(w_{0}, p_{0}\right)+K\left(p_{0}\right)\right]^{-1} \in \operatorname{Lip}\left(L_{2}\left(S^{\prime}\right) \times \mathbb{R}, H_{0}^{2}\right)$

\section{Globale Ergebnisse}

Wir ersetzen die Bedingung $\left(A_{2}\right)$ durch die Bedingung

$\left(\mathrm{A}_{2}\right)(\partial a / \partial t)(s, t) \geq a_{0}>0$ für $(s, t) \in S \times \mathbb{R}$

und leiten einige A-priori-Abschätzungen für Lösungen der Aufgabe (1.8) - (1.10) her.

Lemma 3.1: Unter den Bedingungen $\left(\mathrm{A}_{1}\right),\left(\mathrm{A}_{2}^{s}\right),\left(\mathrm{Q}_{1}\right)-\left(\mathrm{Q}_{3}\right)$ gibt es zu jedem $\mathrm{p}_{\mathrm{O}} \epsilon$ $\mathbb{R}^{+}$und zu jeder Umgebung $U\left(\rho_{0}\right)$ mit kompakter AbschlieBung in $\mathbb{R}^{+}$Konstanten $M_{0}, M_{1}$ derart, daß

$$
0<M_{0} \leq w_{p}^{\prime}(s) \leq M_{1}(s \in S)
$$

fuir alle Lösungen $w_{p} \in W_{0}^{+}$der Gleichung $\mathfrak{F} w_{p}-f(p)=0$ mit $p \in U\left(p_{0}\right)$ gilt. 
Beweis : Sei $w_{p} \in W_{0}^{+}$Lösung der Gleichung $\mathfrak{F} w_{p}-f(p)=0$, d.h. $w_{p} \in W^{+} C$ $W_{2}^{2}\left(S^{\prime}\right)$ genuigt der Gleichung

$$
-d a\left(s, \ln w_{p}^{\prime}(s)\right) / d s+q\left(s, w_{p}(s)-s\right)=0 \text { f.f.a. } s \in S
$$

und den Randbedingungen $w_{p}(0)=0$ und $w_{p}^{\prime}(1)=p$. Für beliebiges $s_{0} \in(0,1]$ ergibt sich daraus

$$
0=\int_{0}^{s_{0}}\left\{-d a\left(s, \ln w_{p}^{\prime}(s)\right) / d s+q\left(s, w_{p}(s)-s\right)\right\}\left(w_{p}(s)-s\right) d s
$$

und weiter

$$
\begin{aligned}
& a\left(s_{0}, \ln w_{p}^{\prime}\left(s_{0}\right)\right)\left(w_{p}\left(s_{0}\right)-s_{0}\right) \\
& =\int_{0}^{s_{0}}\left\{a\left(s, \ln w_{p}^{\prime}(s)\right)\left(\dot{w}_{p}^{\prime}(s)-1\right)+g\left(s, w_{p}(s)-s\right)\left(w_{p}(s)-s\right)\right\} d s .
\end{aligned}
$$

Mit der Darstellung (1.15) erhalten wir speziell für $z_{1}:=w_{p}(s), z_{2}:=1$ unter Benutzung der Eigenschaft $\left(\mathrm{A}_{2}{ }^{s}\right)$

$$
a\left(s, \ln w_{p}^{\prime}(s)\right)\left(w_{p}^{\prime}(s)-1\right) \geq a_{0}\left(w_{p}^{\prime}(s)-1\right) \ln w_{p}^{\prime}(s) \quad(s \in S) .
$$

Berücksichtigen wir noch die Monotonieeigenschaft $\left(\mathrm{Q}_{2}\right)$ der Funktion $q$, so erhalten wir aus den Abschätzungen (3.2), (3.3) die Ungleichungen

$$
w_{p}(s)\left\{\begin{array}{l}
\geq 1 \\
s 1
\end{array} \text { und } w_{p}(s)-s\left\{\begin{array}{ll}
\geq 0 & \text { für } p \geq 1 \\
s 0 & \text { für } p<1
\end{array} \quad(s \in S) .\right.\right.
$$

Dabei sei an die Lösung $w_{1}=$ id erinnert. Für beliebige $s_{0} \in[0,1)$ gilt ferner

$$
0=\int_{s_{0}}^{1}\left\{-d a\left(s, \ln w_{p}^{\prime}(s)\right) / d s+q\left(s, w_{p}(s)-s\right)\right\} d s .
$$

Aus $p \geq 1$ und (3.4) ersehen wir $\int_{s_{0}}^{1} q\left(s, w_{p}(s)-s\right) d s \geq 0$, also

$$
0 \geq \int_{s_{0}}^{1}-d a\left(s, \ln w_{p}^{\prime}(s)\right) \quad \text { bzw. } \quad a\left(s_{0}, \ln w_{p}^{\prime}\left(s_{0}\right)\right) \leq a(1, \ln p) .
$$

Die Bedingung $\left(\mathrm{A}_{2}^{s}\right)$ ergibt dann

$$
a(s, t)=\int_{0}^{\tau}(\partial a / \partial t)(s, \tau) d \tau \geq a_{0} t \quad(t<0 ; s \in S),
$$

schließlich $a_{0} \ln w_{p}^{\prime}\left(s_{0}\right) \leq a(1, \ln p)$ und mit (3.4)

$$
1 \leq w_{p}^{\prime}(s) \leq \exp \left\{a(1, \ln p) / a_{0}\right\} \quad(s \in S) .
$$

Sei nun $0<p<1, s_{0} \in[0,1)$ und folglich nach (3.4) $\int_{s_{0}}^{1} q\left(s, w_{p}(s)^{2}-s\right) d s \leq 0$. Wir ersehen daraus

$$
0 \leq \int_{s_{0}}^{1}-d a\left(s, \ln w_{p}^{\prime}(s)\right)=-a(1, \ln p)+a\left(s_{0}, \ln w_{p}^{\prime}\left(s_{0}\right)\right) \quad\left(s_{0} \in[0,1)\right) .
$$

Für $s \in S$ und $t \leq 0$ gilt 


$$
a(s, t)=-\int_{0}^{|r|}(\partial a / \partial t)(s, \tau) d \tau \leq-a_{0}|t|=a_{0} t,
$$

mit (3.4) also

$$
\exp \left\{a(1, \ln p) / a_{0}\right\} \leq w_{p}(s) \leq 1 \quad(s \in S) .
$$

Aus den Ungleichungen (3.5) für $p \geq 1$ bzw. (3.6) für $0<p<1$ folgt das Lemma

Satz 3.1 : Unter den Bedingungen $\left(\mathrm{A}_{1}\right),\left(\mathrm{A}_{2}{ }^{5}\right),\left(\mathrm{Q}_{1}\right)-\left(\mathrm{Q}_{3}\right)$ ist die Menge M aus (2.1) abgeschlossen.

Beweis : Im Gegensatz zur Behauptung gebe es $z u p_{0} \in \mathbb{R}^{+} \backslash \mathfrak{M}$ eine Folge $\left\{\rho_{n}\right\} \subset \mathbb{N}$ mit $p_{n} \rightarrow p_{0}$. Nach Fixierung der Umgebung $U\left(p_{0}\right)$ können wir für $p_{n} \in U\left(p_{0}\right)$ die Abschätzungen (3.1), für $w_{n}:=w_{P_{n}}$ verwenden. Für alle natürlichen $n, r$ gilt

$$
\begin{aligned}
& \int_{S}\left\{-d\left[a\left(s, \ln w_{n+r}^{\prime}(s)\right)-a\left(s, \ln w_{n}^{\prime}(s)\right)\right] / d s\right. \\
& \left.\quad+q\left(s, w_{n+r}(s)-s\right)-q\left(s, w_{n}\left(s^{\prime}\right)-s\right)\right\}\left(w_{n+r}(s)-w_{n}(s)\right) d s=0 .
\end{aligned}
$$

Mit der Darstellung (1.15) folgt daraus unter Benutzung der Eigenschaften $\left(A_{2}^{s}\right),\left(Q_{2}\right) \operatorname{die}$ Ungleichung

$$
\begin{aligned}
& \left(a\left(1, \ln p_{n+r}\right)-a\left(1, \ln p_{n}\right)\right)\left(w_{n+r}(1)-w_{n}(1)\right) \\
& 2\left(a_{0} / M_{0}\right) \int_{S}\left(w_{n+r}^{\prime}(s)-w_{n}^{\prime}(s)\right)^{2} d s=:\left(a_{0} / M_{0}\right)\left\|w_{n+r}-w_{n}\right\|_{H_{0}^{1}}^{2} .
\end{aligned}
$$

Es ist $\left\{w_{n}\right\} \subset H_{0}^{1}$ demnach Fundamentalfolge und für ein $w_{0} \in H_{0}^{1}$ gilt $\left\|w_{n}-w_{0}\right\|_{w_{2}^{1}} \rightarrow$ 0 . Den Abschätzungen (3.1) für die $w_{n}$ entnehmen wir

$$
0<M_{0} \leq w_{0}^{\prime}(s) \leq M_{1} \text { f.f.a. } s \in S \text {. }
$$

Nach Lemma 1.2 ist der Operator $\Re: W_{2}^{1}\left(S^{\prime}\right) \rightarrow L_{2}\left(S^{\prime}\right),(\Re w)(s)=q(s, w(s)-s)$, Lipschitz-stetig. Dann gilt $\Re w_{n} \rightarrow \Re w_{0}$ in $L_{2}\left(S^{\prime}\right)$. Für $h \in D\left(S^{\prime}\right)$ ist

$$
0=\int_{S} a\left(s, \ln w_{n}^{\prime}(s)\right) h^{\prime}(s) d s+\int_{S} \Re w_{n}(s) h(s) d s .
$$

Aus der Abschätzung (3.7) folgt $a\left(\cdot, \ln w_{0}(\cdot)\right) \in L_{\infty}\left(S^{\prime}\right)$. Die entsprechende Abschätzung (3.1) für die Funktionen $w_{n}$ ermöglicht nun die Anwendung des Lebesgue 'schen Grenzwertsatzes; wir erhalten

$$
0=\int_{S} a\left(s, \ln w_{0}^{\prime}(s)\right) h^{\prime}(s) d s+\int_{S} \Re w_{0}(s) h(s) d s \text { für alle } h \in D\left(S^{\prime}\right) .
$$

Die Funktion $a\left(\cdot, \ln w_{0}(\cdot)\right)$ ist infolgedessen absolutstetig und es gilt

$$
-\dot{d} a\left(s, \ln w_{0}(s)\right) / d s+q\left(s, w_{0}(s)-s\right)=0 \text { f.f.a. } s \in S .
$$

Die entsprechende Gleichung für $w_{n}$ können wir nach Lemma 1.1 in der Form

$$
-\frac{\partial a}{\partial s}\left(s, \ln w_{n}^{\prime}(s)\right)-\frac{\partial a}{\partial t}\left(s, \ln w_{n}^{\prime}(s)\right) \frac{w_{n}^{\prime \prime}(s)}{w_{n}^{\prime}(s)}+q\left(s, w_{n}(s)-s\right)=0 \text { f.f.a. } s \in S
$$

schreiben. Mit den Ungleichungen $(\partial a / \partial t)(s, t) \geq a_{0}>0$ für $(s, t) \in S \times \mathbb{R}$ und $0<M_{0} s$ 
$w_{n}^{\prime}(s) \leq M_{1}$ erhalten wir $\int_{S}\left(w_{n}{ }^{\prime \prime}(s)\right)^{2} d s \leq x$ flir eine von $n \in \mathbb{N}$ unabhängige Konstante $x$. Außerdem ersieht man durch Grenzübergang über Teilfolgen $w_{n}{ }^{\prime \prime}(s) \rightarrow v(\dot{s})$ f.f.a. $s \in S$ und eine gewisse Funktion $v$. Das Lemma von Fatou liefert $v \in L_{2}\left(S^{\prime}\right)$. Sehen wir uns den Grenzübergang genauer an: es ist

$$
w_{n}^{\prime \prime}(s)=\frac{w_{n}^{\prime}(s)}{(\partial a / \partial t)\left(\dot{s}, \ln w_{n}^{\prime}(s)\right)}\left\{q\left(s, w_{n}(s)-s\right)-\frac{\partial a}{\partial s}\left(s, \ln w_{n}^{\prime}(s)\right)\right\}=: v_{n}(s)
$$

f.f.a. $s \in S$. Für alle $h \in D\left(S^{\prime}\right)$ gilt $-\int_{S} w_{n}^{\prime}(s) h^{\prime}(s) d s=\int_{S} v_{n}(s) h(s) d s$, folglich

$$
-\int_{s} w_{0}^{\prime}(s) h^{\prime}(s) d s=\int_{s} v(s) h(s) d s \text {, also } v=w_{0}^{\prime \prime} \in L_{2}\left(S^{\prime}\right) \text {. }
$$

Die Ungleichungen (3.7) liefern zudem $w_{0} \in W^{+}$. Berechnen wir noch den Randwert $w_{0}^{\prime}(1)$. Die Gleichungen

$$
0=-d a\left(s, \ln w^{\prime}(s)\right) / d s+q(s, w(s)-s)=0 \text { f.f.a. } s \in S
$$

für $w=w_{n}(n \in \mathbb{N})$ und $w=w_{0}$ multiplizieren wir mit einer beliebigen Funktion $h \in C^{1}(S)$ mit $h(0)=0$. Daraus ergibt sich

$$
\begin{aligned}
& {\left[a\left(1, \ln p_{n}\right)-a\left(1, \ln w_{0}^{\prime}(1)\right)\right] h(1)} \\
& =\int_{S}\left\{\left[a\left(s, \ln w_{n}(s)\right)-a\left(s, \ln w_{0}(s)\right)\right] h^{\prime}(s)\right. \\
& \left.\quad+\left[g\left(s, w_{n}(s)-s\right)-q\left(s, w_{0}(s)-s\right)\right] h(s)\right\} d s .
\end{aligned}
$$

Der Grenzübergang mit Hilfe des Lebesgue' schen Grenzwertsatzes liefert $a\left(1, \ln w_{0}^{\prime}(1)\right)$ $=a\left(1, \ln p_{0}\right)$. Die Funktion $\cdot a(1, \cdot)$ ist wegen der Bedingung $\left(A_{2}^{s}\right)$ streng monoton wachsend und folglich $w_{0}^{\prime}(1)=p_{0}$, also $p_{0} \in \mathfrak{M}$ im Gegensatz zur Annahme

Da die Menge $\mathbb{R}^{+}$zusammenhängend ist, folgt aus den Sätzen 1.2, 2.1, 3.1 das folgende abschließende Ergebnis.

Satz 3.2: Unter den Bedingungen $\left(A_{1}\right),\left(A_{2}^{s}\right),\left(Q_{1}\right)-\left(Q_{3}\right)$ besitzt die Randwertaufgabe (1.8) - (1.10) für jedes $p \in \mathbb{R}^{+}$genau eine Lösung $w_{p} \in W{ }^{+}$.

\section{LITERATUR}

[1] LANGENBACH, A.: Uber lipschitzstetige implizite Funktionen. Z. Anal. Anw. 8(1988), $289 \cdot 292$.

[2] LANGENBACH, A.: Vorlesungen zur höheren Analysis. Berlin : De. Verlag Wiss. 1984.

[3] VALENT,T.: Boundary Value Problems of Finite Elasticity (Springer Tracts in Natu ral Philosophy: Vol. 31). New York-Berlin-Heidelberg : Springer-Verlag-1988.

Received 02.12.1988, in revised form 03.04.1990

Author's address :

Prof. Dr. Arno Langenbach

Sektion Mathematik der Humboldt-Universität

Unter den Linden 6 ; PF 1297

D (Ost) - 1086 Berlin 